\title{
Radiation-induced pathological fractures of the proximal femur: a case series considering an endoprosthetic solution
}

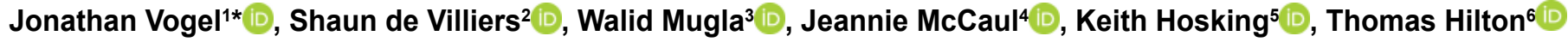 \\ 1 Faculty of Medicine and Health Sciences, University of Cape Town, Cape Town, South Africa \\ 2 Faculty of Medicine and Health Sciences, University of Cape Town, Cape Town, and Frere Hospital, East London, and Faculty of Medicine and Health \\ Sciences, Walter Sisulu University, Mthatha, South Africa \\ 3 Cape Sarcoma Unit, Groote Schuur Hospital, Faculty of Medicine and Health Sciences, University of Cape Town, Cape Town, South Africa \\ 4 Groote Schuur Hospital and Victoria Hospital, Cape Town; Faculty of Medicine and Health Sciences, University of Cape Town, Cape Town, South Africa \\ 5 Vincent Pallotti Life Orthopaedic Hospital, Cape Town; Faculty of Medicine and Health Sciences, University of Cape Town, Cape Town, South Africa \\ 6 Groote Schuur Hospital and Vincent Pallotti Life Orthopaedic Hospital, Cape Town; Faculty of Medicine and Health Sciences, University of Cape Town, \\ Cape Town, South Africa
}

*Corresponding author: jonnovogel@gmail.com

Citation: Vogel J, De Villiers S, Mugla W, McCaul J, Hosking $\mathrm{K}$, Hilton T. Radiation-induced pathological fractures of the proximal femur: a case series considering an endoprosthetic solution. SA Orthop J 2021;20(1):39-42. http://dx.doi. org/10.17159/2309-8309/2021/ v20n1a5

Editor: Prof. Theo le Roux, University of Pretoria, Pretoria, South Africa

Received: June 2020

Accepted: October 2020

Published: March 2021

Copyright: (c) 2021 Vogel J. This is an open-access article distributed under the terms of the Creative Commons Attribution Licence, which permits unrestricted use, distribution and reproduction in any medium, provided the original author and source are credited.

Funding: No funding was received for this study.

Conflict of interest: Authors $\mathrm{KH}$ and $\mathrm{TH}$ are consultants for LRS. The remaining authors declare that they have no conflicts of interest that are directly or indirectly related to this research.

\section{Abstract \\ Background}

Radiation-induced pathological fractures of the proximal femur are difficult to treat due to frequent non-union and hardware failure using standard fracture fixation techniques. This case series investigates endoprosthetic replacement as a treatment option.

\section{Methods}

A retrospective folder review from a private hospital in Cape Town, of patients who had sustained a radiation-induced pathological fracture, was reviewed using descriptive statistics.

\section{Results}

Six patients met the inclusion criteria. One patient was excluded as the minimum follow-up time of six months was not met. Of the five patients that were analysed, all five sustained transverse, subtrochanteric femur fractures. Prior to definitive treatment with a proximal femoral replacement, three patients were treated with standard trauma instrumentation prior to referral to the unit, and one patient was treated with a vascularised fibular graft as their initial treatment while at the unit. One patient was treated with an endoprosthetic replacement as their first procedure at the unit. Among the three patients treated with standard trauma fixation and the one patient treated with a vascularised fibular graft, there was a $100 \%$ failure rate. One standard trauma instrumentation patient had an ablation due to free musculocutaneous flap failure and periprosthetic infection after endoprosthetic replacement. This was the only complication of endoprosthetic replacement. At a median follow-up of 15 months ( $\min 7, \max 55)$ the median Musculoskeletal Tumour Society score was $74 \%(\min 63 \%$, $\max 93 \%)$.

\section{Conclusion}

This case series seeks to highlight the high failure rates seen when treating this condition with standard trauma instrumentation or biological methods. Further research is needed, but endoprosthetic replacement may be a viable alternative solution.

Level of evidence: Level 4

Keywords: radiation-induced fracture, pathological fracture, endoprosthetic replacement 


\section{Introduction}

External beam radiation therapy (EBRT) is frequently used to treat soft tissue sarcomas. It may cause significant side effects to the adjacent skeleton including osteomyelitis, growth plate arrest and osteonecrosis. ${ }^{1-4}$ The proximal femur is vulnerable to these complications during EBRT for tumours of the thigh and pelvis. Osteonecrosis after EBRT may result in a pathological fracture of the proximal femur, which has a reduced ability to heal and remodel due to the high stressors during weight bearing and poor blood supply in this area. In our experience, a transverse pathological fracture typically occurs at the subtrochanteric region.

Figure 1 demonstrates the macro- and microscopic differences between fat necrosis in marrow caused by EBRT and normal marrow.

The healing potential of these fractures is markedly impaired. ${ }^{5}$ Multiple studies have shown that treating these fractures with standard trauma fixation methods results in failure rates as high as $63 \%$ to $82 \%$. $^{4-6}$ These non-unions are so refractory to treatment that in a study by Sternheim et al., $46 \%$ of patients required more than one revision procedure. ${ }^{5}$ Because of the poor outcomes associated with standard methods of fixation, multiple authors have opted for more aggressive surgical management of these fractures. These include combined vascularised fibula autograft and plating, ${ }^{7}$ vascularised periosteal free-flap graft ${ }^{8}$ and prophylactic intramedullary nailing of patients at high risk of fracture (Table I). ${ }^{9-11}$

Kim et al. demonstrated a low complication rate with the use of primary prosthetic joint replacement. They showed a statistically significant improvement in time to mobilisation, and a decrease in the incidence of complications and re-operations when compared to standard fixation methods. ${ }^{4}$ Mavrogenis et al. confirmed this with his own study using primary prosthetic joints to treat these fractures. ${ }^{12}$ Both authors suggested further investigation of primary joint replacement as a treatment option for radiotherapyinduced pathological fractures of the femur.

Due to the absence of level 1 and 2 studies, Soares et al. could not determine the most effective and safest method of treatment in their recent systematic review. They highlighted the importance of understanding that these fractures have poor longterm outcomes when treated with standard methods of internal fixation. ${ }^{1}$

We describe a case series of patients who were referred to our unit after failing treatment for their radiation-induced pathological fracture using standard fracture fixation and/or biological reconstructive techniques. Our aim is to highlight this diagnosis and the difficulty in its management using standard fracture fixation principles.

\section{Materials and methods}

This study was conducted after institutional review board and ethical approval. A retrospective folder review was conducted between 1 January 2012 and 31 January 2016. The criterion used to select patients for inclusion in the folder review was a diagnosis of a radiation-induced pathological fracture of their proximal femur. This diagnosis was made in patients with a history of EBRT to the pelvis or proximal thigh, and imaging consistent with a pathological fracture. The criteria for excluding patients were: missing data, less than six months of follow-up and whether the fracture was secondary to metastases or trauma.

We recorded the following: patient age, tumour type, radiotherapy dose, time from diagnosis to fracture, time from fracture to endoprosthetic replacement (EPR), number of surgeries, followup time, complications requiring surgery and Musculoskeletal Tumour Society (MSTS) score.

Descriptive statistics were used to present the results.
Table I: Risk factors for radiation-associated fracture , $^{1,9-11}$

\begin{tabular}{|l|l|}
\hline & \multicolumn{1}{c|}{ Risk factor } \\
\hline 1 & Larger tumour size \\
\hline 2 & Advanced age \\
\hline 3 & Female sex \\
\hline 4 & Tumour located in anterior compartment of thigh \\
\hline 6 & Periosteal stripping at time of tumour resection \\
\hline 6 & High dose radiotherapy (controversial) \\
\hline
\end{tabular}

\section{Results}

Six patients met the inclusion criteria and one was excluded as the minimum follow-up time was not met. The results are shown in Table II. Three of the patients (patients A, B and C) had EBRT as adjuvant treatment after resection of a soft tissue sarcoma from the proximal thigh, while two patients (patients $D$ and $E$ ) had EBRT for proximal femoral breast metastases prior to any surgical intervention. These metastases did not cause a pathological fracture themselves but rather the radiotherapy used to treat the lesions caused a radiation-induced fracture. All five patients sustained a transverse subtrochanteric proximal femur fracture (Figure 2). Three of the five patients (patients C, D and E) were referred to us after multiple failed trauma fixation attempts using standard instrumentation (Figure 3 and 4). This failed in all three patients due to continued non-union of the fracture. Patient $\mathrm{C}$
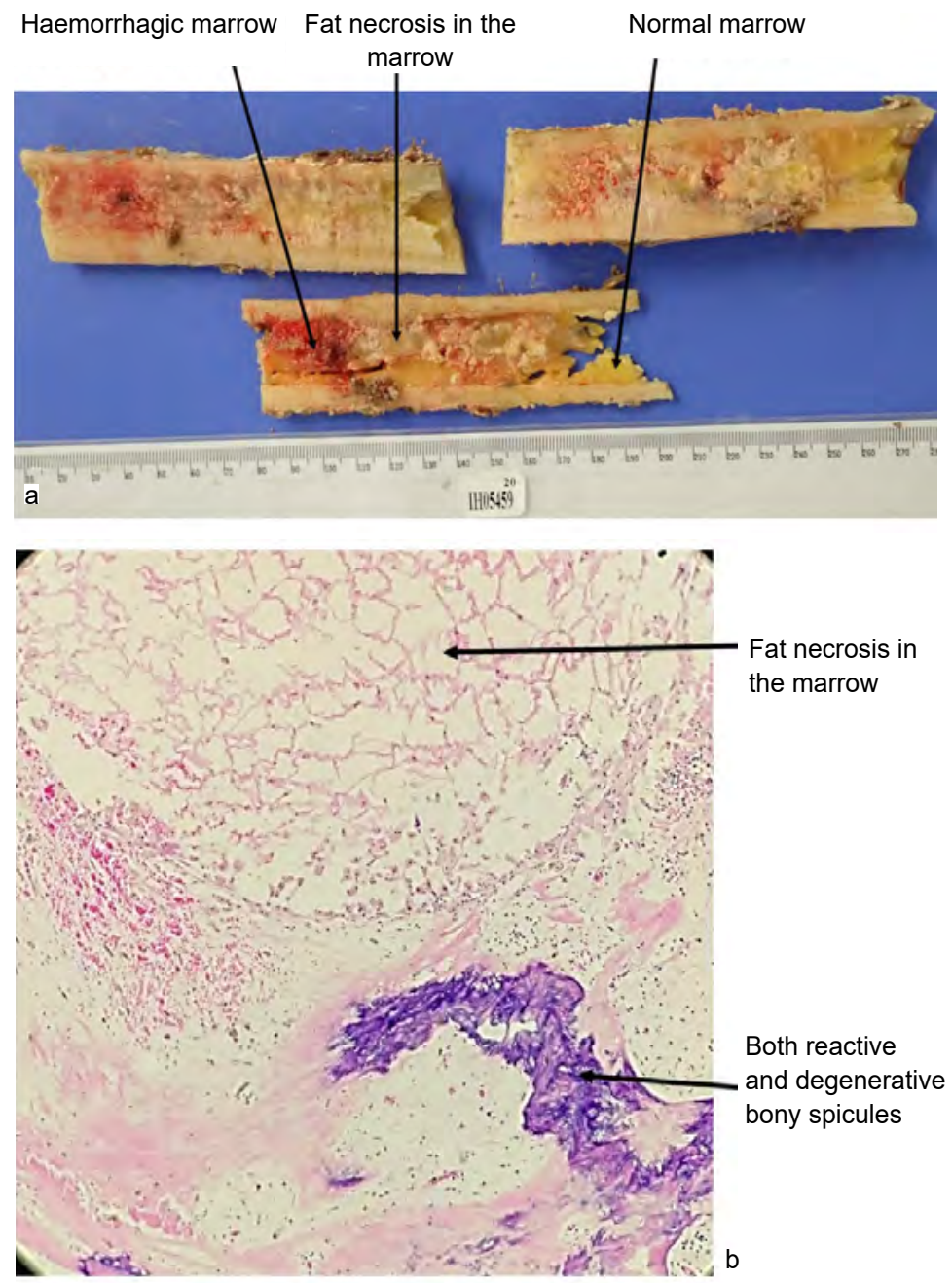

Figure 1a and b. Demonstrating EBRT-induced fat necrosis macroscopically and microscopically, respectively 
Table II: Patient results

\begin{tabular}{|c|c|c|c|c|c|c|c|c|}
\hline Patient & $\begin{array}{c}\text { Age } \\
\text { (years) }\end{array}$ & Sex & $\begin{array}{c}\text { Interval from } \\
\text { diagnosis to } \\
\text { fracture (months) }\end{array}$ & $\begin{array}{l}\text { Interval from } \\
\text { fracture to EPR } \\
\text { (months) }\end{array}$ & $\begin{array}{l}\text { Radiation dose } \\
\text { (Gy) }\end{array}$ & $\begin{array}{l}\text { Number of } \\
\text { surgeries }\end{array}$ & $\begin{array}{c}\text { Follow-up } \\
\text { (months) }\end{array}$ & $\begin{array}{c}\text { MSTS score } \\
(\%)\end{array}$ \\
\hline A & 46 & $\mathrm{~F}$ & 180 & 69 & 66 & 3 & 30 & 63 \\
\hline B & 63 & $\mathrm{~F}$ & 40 & 1 & 66 & 1 & 55 & 93 \\
\hline C & 66 & $M$ & 111 & 43 & 66 & 3 & 12 & n/a (ablation) \\
\hline $\mathrm{D}$ & 65 & $\mathrm{~F}$ & 208 & 41 & 20 & 3 & 7 & 80 \\
\hline$E$ & 45 & $\mathrm{~F}$ & 27 & 5 & 20 & 3 & 15 & 67 \\
\hline Median & 63 & & 111 & 41 & 66 & 3 & 15 & 73.5 \\
\hline
\end{tabular}

failed two intramedullary (IM) nails, patient $D$ failed an IM nail followed by a sliding hip screw and patient $E$ failed an IM nail. After referral to our unit, all three patients (patients $C, D$ and $E$ ) were then converted to an endoprosthetic replacement (EPR) (Figure 5). Two patients were referred to us prior to any fixation attempts (patients $A$ and $B$ ). Patient $A$ underwent a vascularised fibular graft and plating, which failed to incorporate. This was then converted to an EPR. Patient B had an EPR performed as the primary surgery. At final follow-up the median MSTS score was $74 \%$ ( $\min 63 \%$, max 93\%). The patient who had an EPR as their initial treatment had an MSTS score of $93 \%$.

One of the patients (patient C) who failed standard fixation techniques required a free musculocutaneous flap to cover the EPR due to poor soft tissue after EBRT and repeated surgeries. The flap failed and dehisced resulting in a peri-prosthetic joint infection (PJI). The patient chose amputation as their treatment option. There were no other complications noted after EPR in the other four patients.

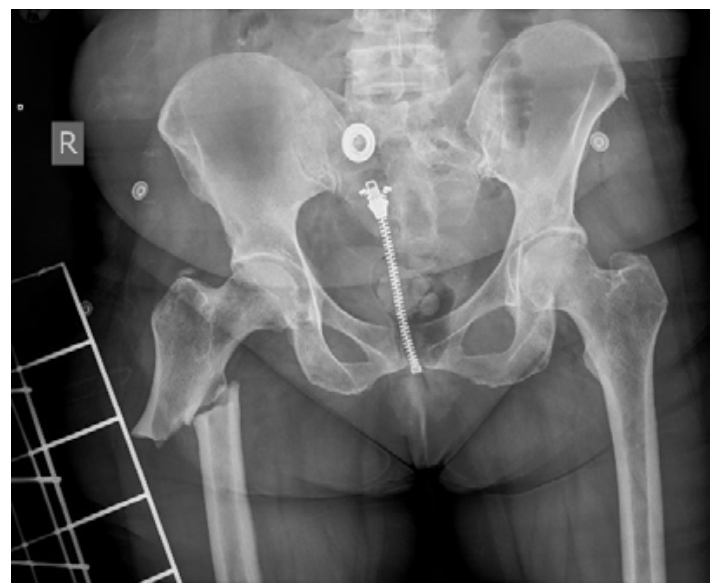

Figure 2. Antero-posterior (AP) pelvis X-ray demonstrating a radiationinduced pathological fracture of the proximal femur. Note the transverse nature of the fracture, adjacent sclerosis and its subtrochanteric location.

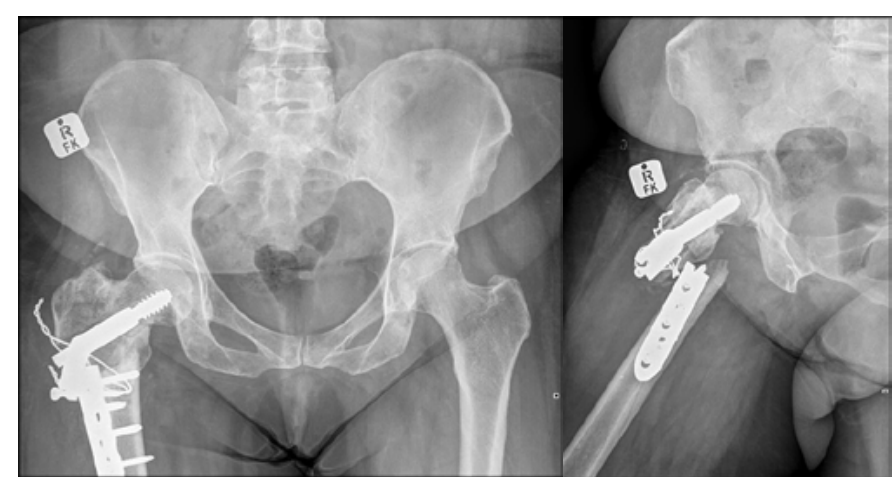

Figure 3. AP and lateral X-rays showing failure of dynamic hip screw of the same patient (patient D)

\section{Discussion}

The incidence of radiation-induced pathological fractures is unknown but thought to be rare. ${ }^{4}$ However, Elliot et al. showed that pelvic irradiation increases the incidence of proximal femur fractures by up to $76 \% .{ }^{2}$ Therefore, it is important to be alert to the possibility of this diagnosis in patients at high risk (Table $I$ ) who present with a fracture. Although we cannot quantify this statistically due to the rarity of this condition, it is our experience that pain is the main symptom that precedes or identifies an impending radiationinduced pathological fracture and can be useful in its diagnosis and treatment. Our results are in keeping with the current literature which holds that standard fracture fixation methods and biological reconstruction techniques have an unacceptably high failure rate. All four patients who were not treated primarily with an EPR had failures of instrumentation, multiple revision surgeries and required an EPR as their definitive treatment. The decision to perform an EPR first has been suggested to deliver a more predictable outcome and reduce the need for repeat surgeries. ${ }^{4}$ This is consistent with our series, as the only patient to have an EPR as their initial fracture treatment only required one surgical procedure and had the highest MSTS score of 93\%. Having an EPR first may prevent ongoing morbidity and cost to the patient, and maximise their quality of life and function. The median time of 41 months from fracture to final surgery and total number of surgeries, median 3 , in our series highlights the impact this may have on the patient.

EPR however, is not without its own set of significant complications, one of which is infection. In our series, we had one case of deep infection. The patient had received significant radiotherapy of $66 \mathrm{~Gy}$ to the proximal thigh and had two previous surgeries using standard trauma instrumentation which failed to induce union of their fracture. As a result, the soft tissues were severely compromised and required a free musculocutaneous flap to cover the definitive proximal femoral replacement. The flap, however, failed, which led to a PJI, and an ablation of the limb was performed. The patient chose this treatment option over a staged revision of his EPR. We

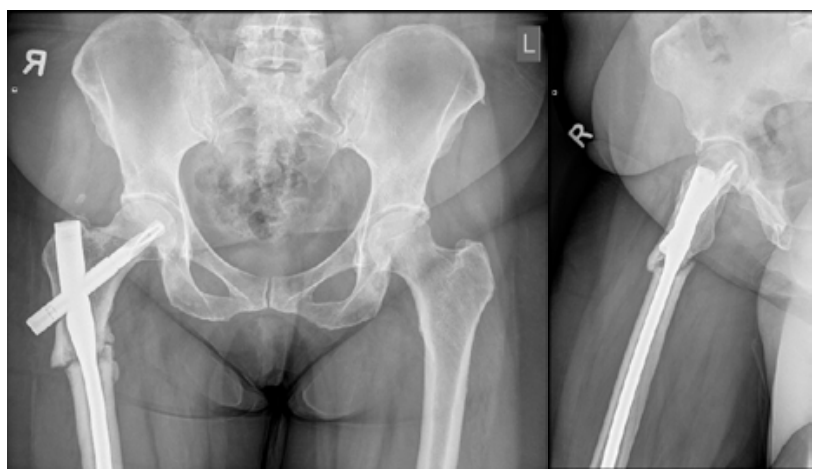

Figure 4. AP and lateral X-rays of fractured intramedullary nail inserted into the patient (patient D) 


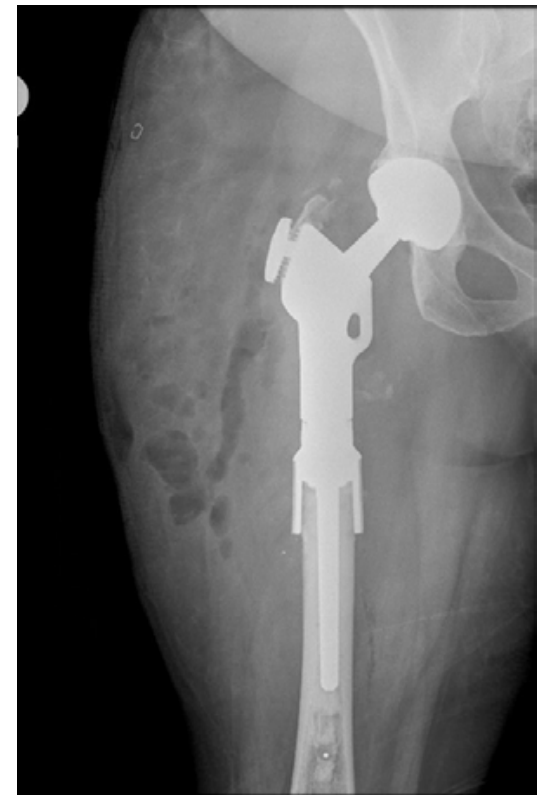

Figure 5. AP X-ray after endoprosthetic replacement of the proximal femur

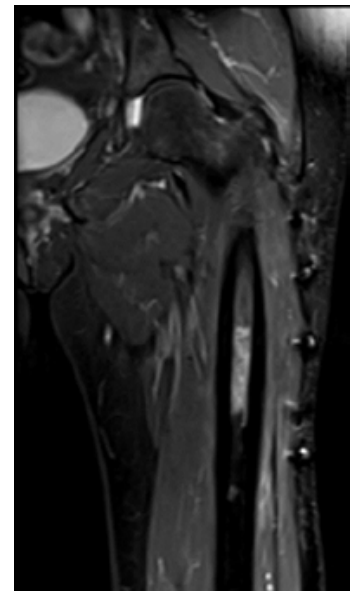

Figure 6. MRI T1 TRIM COR showing a segment of medullary hyperintensity in the proximal femur. Report noted sharply defined proximal and distal margins with no cortical expansion or disruption

regard this as due to failure of soft tissue cover rather than the use of an EPR.

Biological methods of treatment of radiation-induced pathological fractures have been described in the literature. One such method includes using a vascularised fibula autograft to reconstruct the femoral defect after excision of the pathological fracture. ${ }^{7}$ This was attempted in one of our patients but it failed to incorporate, possibly due to the underassessment of the extent of devascularised bone. This under-resection occurred despite $7 \mathrm{~cm}$ of femur, including the fracture site, being resected to healthy bleeding bone. This patient had required radiation to a large area of the proximal thigh, which resulted in extensive avascular necrosis (AVN) of the femur. It is our view that the degree of AVN in this scenario is difficult to assess using advanced imaging such as MRI (Figure 6) or by intraoperative observations.

Many cases of radiotherapy-induced pathological fracture are mislabelled as pathological fractures due to metastases. ${ }^{3}$ This is because differentiating between the two types of fracture on modern imaging can be difficult, but it is important to do so as the treatments are different for both and unnecessary investigations and treatments must be avoided. It has also been our observation that radiation-induced AVN may show cortical scalloping and intracortical collections that may be misdiagnosed as chronic osteitis on MRI scans by reporting radiologists. This may mislead the treating surgeon and result in the incorrect management of the patient.

We acknowledge several limitations of our study. These include the small number of patients which makes statistical analysis difficult. Our follow-up period is also short to medium term and therefore there may be late complications of EPR that are not included in this series, such as aseptic loosening. Longer followup is needed. We have presented our case series, not to make definitive conclusions, but to highlight the difficulties surgeons face when treating patients with this problem. Our results match those reported in the current literature and suggest that more aggressive treatment of these fractures with EPR may provide a more predictable outcome for the patient.

\section{Conclusion}

Radiation-induced pathological fractures are notoriously difficult to treat and can lead to multiple surgeries, severe morbidity and

prolonged hospitalisation. In patients with a history of EBRT to the pelvis or proximal thigh, medical practitioners need to be highly alert to this condition. Our case series, as well as the literature reviewed, suggest that a more aggressive treatment with primary EPR may provide a more predictable outcome for the patient.

\section{Ethics statement}

For this retrospective study formal consent was not required. Ethical approval number HREC 889/2019

The author/s declare that this submission is in accordance with the principles laid down by the Responsible Research Publication Position Statements as developed at the 2nd World Conference on Research Integrity in Singapore, 2010.

\section{Declaration}

The authors declare authorship of this article and that they have followed sound scientific research practice. This research is original and does not transgress plagiarism policies

\section{Author contributions}

JV: Study design, data collection, data analysis, manuscript preparation

SD: Study design, data analysis, manuscript preparation

WM: Data analysis, data collection, manuscript preparation

JM: Data analysis, manuscript preparation

TH: Study conceptualisation and design, data collection and analysis, manuscript preparation

$\mathrm{KH}$ : Study conceptualisation and design as well as data collection and analysis

\section{References}

1. Soares BCG, De Araujo ID, Pádua BJ, et al. Pathological fracture after radiotherapy: systematic review of literature. Rev Assoc Med Bras. 2019;65(6):902-908. https://doi.org/10.1590/1806-9282.65.6.902.

2. Elliott SP, Jarosek SL, Alanee SR, et al. 3-Dimensional external beam radiotherapy for prostate cancer increases the risk of hip fracture. Cancer. 2011;117(19):612-26. https://doi.org/10.1002/cncr.25994.3.

3. Iğdem S, Alço G, Ercan T, et al. Insufficiency fractures after pelvic radiotherapy in patients with prostate cancer. Int J Radiat Oncol Biol Phys. $2010 \mathrm{Jul}$ 1;77(3):818-23. https://doi.org/10.1016/j.jijrobp.2009.05.059.

4. Kim HJ, Healey JH, Morris CD, Boland PJ. Site-dependent replacement or internal fixation for postradiation femur fractures after soft tissue sarcoma resection. Clin Orthop Relat Res. 2010;468:3035-40. https://doi.org/10.1007/ s11999-010-1405-6.

5. Sternheim A, Saidi K, Lochab J, et al. Internal fixation of radiation-induced pathological fractures of the femur has a high rate of failure. Bone Joint $\mathrm{J}$. 2013;95(8):1144-48. https://doi.org/10.1302/0301-620X.95B8.31832.

6. Helmstedter CS, Goebel M, Zlotecki R, Scarborough MT. Pathologic fractures after surgery and radiation for soft tissue tumors. Clin Orthop Relat Res. 2001;(389):165-72.

7. Duffy G, Wood M, Rock M, Sim F. Vascularized free fibular transfer combined with autografting for the management of fracture nonunions associated with radiation therapy. J Bone Jt Surg. 2000;82(4):544-54. https://doi. org/10.2106/00004623-200004000-00009.

8. Fuchs B, Steinmann SP, Bishop AT. Free vascularized corticoperiosteal bone graft for the treatment of persistent nonunion of the clavicle. J Shoulder Elb Surg. 2005;14(3):264-68. https://doi.org/10.1016/j.jse.2004.06.007.

9. Gortzak Y, Lockwood GA, Mahendra A, Wang Y. Prediction of pathologic fracture risk of the femur after combined modality treatment of soft tissue sarcoma of the thigh. Cancer. 2010;116(6):1553-59. https://doi.org/10.1002/ cncr.24949.

10. Cannon C, Lin P, Lewis VO, Yasko AW. Management of radiation-associated fractures. Am Acad Orthop Surg. 2008;16(9):541-49.

11. Lin PP, Schupak KD, Boland PJ, Brennan MF, Healey JH. Pathologic femoral fracture after periosteal excision and radiation for the treatment of soft tissue sarcoma. Cancer. 1998;(82):2356-65

12. Mavrogenis A, Pala E, Romantini M, Guerra G, et al. Side effects of radiation in musculoskeletal oncology: clinical evaulation of radiationinduced fractures. Int J Immunopathol Pharmacol. 2011;24:29-37. https://doi. org/10.1177/03946320110241S207. 